PROCEEDINGS OF THE

AMERICAN MATHEMATICAL SOCIETY

Volume 127, Number 6, Pages 1771-1780

S 0002-9939(99)05115-1

Article electronically published on February 11, 1999

\title{
BIMODULES OVER NEST ALGEBRAS AND DEDDENS' THEOREM
}

\author{
I. TODOROV
}

(Communicated by David R. Larson)

\begin{abstract}
We generalize Deddens' theorem for nest algebras in the case of $\mathrm{w}^{*}$-closed nest algebras bimodules. For each such bimodule, we introduce a norm closed sub-bimodule of it, which corresponds to the radical of a nest algebra and describe it in a number of ways, generalizing known facts about nest algebras.
\end{abstract}

\section{INTRODUCTION}

If $\mathcal{H}_{1}$ and $\mathcal{H}_{2}$ are complex Hilbert spaces, $\mathcal{B}\left(\mathcal{H}_{1}, \mathcal{H}_{2}\right)$ will denote the algebra of all bounded linear operators from $\mathcal{H}_{1}$ to $\mathcal{H}_{2}$. In the case $\mathcal{H}_{1}=\mathcal{H}_{2}=\mathcal{H}$, we write $\mathcal{B}(\mathcal{H})$. Given a set $\mathcal{L}$ of (closed) subspaces of a Hilbert space $\mathcal{H}$, by $\operatorname{Alg} \mathcal{L}$ is denoted as usual the algebra of all operators on $\mathcal{H}$, which leave each element of $\mathcal{L}$ invariant. Dually, if $\mathcal{S}$ is a set of operators, by Lat $\mathcal{S}$ we will mean the (complete) lattice of (closed) subspaces, which are left invariant by every element of $\mathcal{S}$. If $T$ is a positive invertible operator in $\mathcal{B}(\mathcal{H})$ and $E_{T}$ its spectral measure, by the spectral nest of $T$ we will mean the nest $\mathcal{E}_{T}=\left\{E_{t}\right\}_{t>0}$, where $E_{t}=E_{T}[0, t]$. Let $\mathcal{N}$ be a (complete) nest of (closed) subspaces of $\mathcal{H}$ and $\mathcal{A}=\operatorname{Alg} \mathcal{N}$ be the corresponding nest algebra. In [2] Deddens proved that a necessary and sufficient condition for an operator $X$ to belong to the nest algebra $\mathcal{A}$ is that $\left\|A^{n} X A^{-n}\right\|$ be bounded in $n$ where $A$ is a positive invertible operator having spectral nest $\mathcal{N}$. He used results of Loebl and Muhly [9]. Elementary proofs of this result were given by Radjabalipour [11] and Erdos [3]. The approach of Erdos allowed him to describe the radical of a nest algebra in terms analogous to those in Deddens' theorem. Namely, if $\mathcal{A}_{0}$ is the set of the invertible positive contractions with spectral nest $\mathcal{N}$, and if, for each $A \in \mathcal{A}_{0}$, $\mathcal{R}_{A}$ denotes the set $\left\{X \in \mathcal{B}(\mathcal{H}):\left\|A^{n} X A^{-n}\right\| \longrightarrow_{n} 0\right\}$, he proved that

$$
\operatorname{rad} \mathcal{A}=\overline{\bigcup\left\{\mathcal{R}_{A}: A \in \mathcal{A}_{0}\right\}},
$$

the closure being taken in the norm topology. This paper is an attempt to generalize these results to nest algebra bimodules.

Let $\mathcal{H}_{1}$ and $\mathcal{H}_{2}$ be complex Hilbert spaces and $A$ and $B$ be positive invertible contractions on $\mathcal{H}_{1}$ and $\mathcal{H}_{2}$, respectively. Let

$$
\mathcal{B}_{A, B}=\left\{X \in \mathcal{B}\left(\mathcal{H}_{1}, \mathcal{H}_{2}\right):\left\|B^{n} X A^{-n}\right\| \text { is bounded in } n\right\} .
$$

Received by the editors September 16, 1997.

1991 Mathematics Subject Classification. Primary 47D15; Secondary 47D25.

Key words and phrases. Bimodule, nest algebra, spectral nest, Deddens' theorem.

This work was supported by a grant of the Greek State Scholarship Foundation. 
We show that $\mathcal{B}_{A, B}$ is a (reflexive) nest algebra bimodule. Conversely, given any $\mathrm{w}^{*}$ closed nest algebra bimodule $\mathcal{U}$, for each operator $A$ in a certain class of operators $\mathcal{A}_{1}$ on $\mathcal{H}_{1}$ (for exact definition see the notes after Theorem 2.1), there exist an operator $B_{A}$ on $\mathcal{H}_{2}$, such that

$$
\mathcal{U}=\mathcal{B}_{A, B_{A}} .
$$

Using the Ringrose condition [12] we define a norm closed submodule $\mathcal{R}$ of $\mathcal{U}$ which generalizes the Jacobson radical of a nest algebra. If

$$
\mathcal{R}_{A}=\left\{X \in \mathcal{B}\left(\mathcal{H}_{1}, \mathcal{H}_{2}\right):\left\|B_{A}^{n} X A^{-n}\right\| \longrightarrow 0\right\}
$$

we prove that

$$
\mathcal{R}=\overline{\bigcup\left\{\mathcal{R}_{A}: A \in \mathcal{A}_{1}\right\}} .
$$

We also give other equivalent characterisations of the bimodule $\mathcal{R}$, which generalize known facts about nest algebras. Finally, we identify the maximal $\mathrm{w}^{*}$-closed bimodules of $\mathcal{U}$ and prove that their intersection equals the $\mathrm{w}^{*}$-closure of $\mathcal{R}$.

\section{DedDens' THEOREM FOR NEST ALGEBRA BIMODUleS}

Let $\mathcal{H}_{1}$ and $\mathcal{H}_{2}$ be Hilbert spaces. If $x \in \mathcal{H}_{2}$ and $y \in \mathcal{H}_{1}$ are vectors, by $x \otimes y$ will be denoted the rank one operator from $\mathcal{H}_{1}$ to $\mathcal{H}_{2}$ given by $(x \otimes y)(z)=(z, y) x$. If $\mathcal{S}$ is a subspace and $\mathcal{A}$ and $\mathcal{B}$ are algebras of operators, we say that $\mathcal{S}$ is a $\mathcal{B}, \mathcal{A}$ bimodule, if $\mathcal{B S A} \subseteq \mathcal{S}$. We call a subspace $\mathcal{S}$ of operators reflexive, if $\mathcal{S}=\{T \in$ $\mathcal{B}\left(\mathcal{H}_{1}, \mathcal{H}_{2}\right): T x \in \overline{\mathcal{S} x}$, for every vector $\left.x \in \mathcal{H}_{1}\right\}$ [13]. We recall some definitions from [4] and [5]. A positive operator $T$ with spectral nest $\mathcal{E}_{T}=\left\{E_{t}\right\}$ is said to have a jump at a projection $E$, if there exists an interval $(a, b)$ of the real line, such that $E=E_{t}$ for each $t \in(a, b)$. Next, if $\mathcal{P}_{1}$ and $\mathcal{P}_{2}$ are the lattices of all projections on the Hilbert spaces $\mathcal{H}_{1}$ and $\mathcal{H}_{2}$, respectively, we write $\mathcal{M}\left(\mathcal{P}_{1}, \mathcal{P}_{2}\right)$ for the set of those mappings from $\mathcal{P}_{1}$ to $\mathcal{P}_{2}$ that are join-continuous and zero-preserving. As was proved in [5], each element $\varphi$ of $\mathcal{N}\left(\mathcal{P}_{1}, \mathcal{P}_{2}\right)$ gives rise to two semi-sublattices $\mathcal{L}_{1}, \mathcal{L}_{2}$ of $\mathcal{P}_{1}$ and $\mathcal{P}_{2}$, such that $\mathcal{L}_{1}$ is meet-complete and contains the identity, $\mathcal{L}_{2}$ is join-complete and contains the zero element, and $\varphi_{0}=\varphi \mid \mathcal{L}_{1}: \mathcal{L}_{1} \longrightarrow \mathcal{L}_{2}$ is an order-preserving bijection. Conversely, each order-preserving bijection between two semi-lattices $\mathcal{L}_{1}$ and $\mathcal{L}_{2}$ with the above properties, extends uniquely to some element $\varphi$ of $\mathcal{M}\left(\mathcal{P}_{1}, \mathcal{P}_{2}\right)$. Those $\varphi \in \mathcal{M}\left(\mathcal{P}_{1}, \mathcal{P}_{2}\right)$, for which the respective semi-lattices are totally ordered, will be called nest mappings. Given a set $\mathcal{S}$ of operators, by MapS will be denoted [5] the mapping which sends a projection $P$ to the projection onto the subspace $\bigvee\{S P: S \in \mathcal{S}\}$. As is proved in [5], this mapping belongs to $\mathcal{M}\left(\mathcal{P}_{1}, \mathcal{P}_{2}\right)$. Dually, given any mapping $\varphi$ from $\mathcal{P}_{1}$ to $\mathcal{P}_{2}$, the space $\mathrm{Op} \varphi$ is defined as the collection of operators from $\mathcal{H}_{1}$ to $\mathcal{H}_{2}$, which map the subspace $P \mathcal{H}_{1}$ of $\mathcal{H}_{1}$ into the subspace $\varphi(P) \mathcal{H}_{2}$ of $\mathcal{H}_{2}$ for each projection $P$ in $\mathcal{P}_{1}$. A theorem of Erdos [5] states that $\mathrm{Op} \varphi=\left\{X \in \mathcal{B}\left(\mathcal{H}_{1}, \mathcal{H}_{2}\right): X P \subseteq \varphi(P)\right.$ for each $\left.P \in \mathcal{L}_{1}\right\}$. Also, a subspace $\mathcal{S}$ of operators is reflexive if and only if it is of the form $\mathcal{S}=\operatorname{Op} \varphi$ for some mapping $\varphi$. Let $\mathcal{N}_{1}$ and $\mathcal{N}_{2}$ be nests on the Hilbert spaces $\mathcal{H}_{1}$ and $\mathcal{H}_{2}$ and let $\mathcal{A}=\operatorname{Alg} \mathcal{N}_{1}$ and $\mathcal{B}=\operatorname{Alg} \mathcal{N}_{2}$ be the corresponding nest algbras. The following result of Erdos and Power [7] will be of importance to us.

Theorem 2.1. Let $\mathcal{U}$ be a $\mathcal{B}, \mathcal{A}$-bimodule, closed in any of the weak, ultraweak, strong or ultrastrong topologies. Then there exists a left-continuous order-preserving mapping $\psi$ from $\mathcal{N}_{1}$ to $\mathcal{N}_{2}$ with $\psi(0)=0$, such that $\mathcal{U}=O p \psi$. In fact, $\psi=$ Map $\mathcal{U}$. 
Left-continuity is the property $\lim _{E \uparrow F} \psi(E)=\psi\left(\lim _{E \uparrow F} E\right)$. In the present (totally ordered) case, this notion coincides with join-continuity.

Now let $A$ and $B$ be positive invertible contractions on $\mathcal{H}_{1}$ and $\mathcal{H}_{2}$, respectively, and let $\mathcal{N}_{1}=\mathcal{E}_{A}=\left\{E_{\lambda}\right\}$ and $\mathcal{N}_{2}=\mathcal{E}_{B}=\left\{F_{\lambda}\right\}$ be the corresponding spectral nests. Let $\mathcal{A}=\operatorname{Alg} \mathcal{N}_{1}$ and $\mathcal{B}=\operatorname{Alg} \mathcal{N}_{2}$. As was mentioned, by $\mathcal{B}_{A, B}$ we denote the set $\left\{X \in \mathcal{B}\left(\mathcal{H}_{1}, \mathcal{H}_{2}\right): \sup _{n}\left\|B^{n} X A^{-n}\right\|<\infty\right\}$. First we prove the following theorem.

Theorem 2.2. $\mathcal{B}_{A, B}$ is a (reflexive) $\mathcal{B}, \mathcal{A}$-bimodule. In fact,

$$
\mathcal{B}_{A, B}=\left\{X \in \mathcal{B}\left(\mathcal{H}_{1}, \mathcal{H}_{2}\right):\left(I-F_{\lambda}\right) X E_{\lambda}=0 \text {, for each } \lambda, 0 \leq \lambda \leq 1\right\} .
$$

Proof. The proof of this theorem was inspired by the proof of Theorem 1 of [3]. We first prove the equality of the the two sets. Let the operator $X$ map the subspace $E_{\lambda} \mathcal{H}_{1}$ into the subspace $F_{\lambda} \mathcal{H}_{2}$ for every $\lambda$ between zero and one. For a fixed $\lambda$, write $E=E_{\lambda}, F=F_{\lambda}$. Let $S_{E, \alpha}=\alpha E+I-E$ and $T_{F, \alpha}=\alpha F+I-F$ for $0<\alpha \leq 1$. We define $g(\alpha)=F X E+(I-F) X(I-E)+\alpha F X(I-E)$ for $0 \leq \alpha \leq 1$. As is easily seen, $\|g(0)\| \leq\|X\|$ and $\|g(1)\| \leq\|X\|$, so by convexity of the norm, $\|g(\alpha)\| \leq\|X\|$ for each $\alpha, 0 \leq \alpha \leq 1$. But if $\alpha>0, S_{E, \alpha}$ is invertible $\left(S_{E, \alpha}^{-1}=\alpha^{-1} E+I-E\right)$ and $g(\alpha)=T_{F, \alpha} X S_{E, \alpha}^{-1}$. So for each $S$ and $T$ of the form $S=S_{E, \alpha}, T=T_{F, \alpha}$, we have that $\left\|T X S^{-1}\right\| \leq\|X\|$. Because $T X S^{-1}$ also maps the projection $E_{\lambda}$ into $F_{\lambda}$ for each $\lambda$, it follows by induction that

$$
\left\|\left(\prod T_{k}\right) X\left(\prod S_{k}\right)^{-1}\right\| \leq\|X\|
$$

for each finite set $S_{k}$ and $T_{k}$ of operators of the above form. Now we can choose finite families $\left\{S_{k}\right\}$ such that

$$
\prod S_{k} \longrightarrow A
$$

Of course this implies that

$$
\prod T_{k} \longrightarrow B
$$

which gives

$$
\left\|B X A^{-1}\right\| \leq\|X\| .
$$

Again by induction, we obtain

$$
\left\|B^{n} X A^{-n}\right\| \leq\|X\|
$$

for each $n=1,2, \ldots$

Now let $X \in \mathcal{B}_{A, B}$. We want to show that $\left(I-F_{\lambda}\right) X E_{\lambda}=0$ for each $\lambda, 0 \leq \lambda \leq 1$. Let $\lambda$ be fixed and $\mu>\lambda$. We have

$$
\left\|\left(I-F_{\mu}\right) X E_{\lambda}\right\| \leq\left\|\left(I-F_{\mu}\right) B^{-n}\right\|\left\|B^{n} X A^{-n}\right\|\left\|A^{n} E_{\lambda}\right\| .
$$

Obviously $\left\|A^{n} E_{\lambda}\right\| \leq \lambda^{n}$ and $\left\|\left(I-F_{\mu}\right) B^{-n}\right\| \leq \mu^{-n}$. This means that

$$
\left\|\left(I-F_{\mu}\right) X E_{\lambda}\right\| \leq c\left(\frac{\lambda}{\mu}\right)^{n},
$$

where $c$ is an upper bound for the set $\left\{\left\|B^{n} X A^{-n}\right\|: n=1,2, \ldots\right\}$. Letting $n \longrightarrow \infty$ in the above inequality, we get

$$
\left(I-F_{\mu}\right) X E_{\lambda}=0
$$

for each $\mu>\lambda$. Since $E_{\lambda}=\lim _{\mu \downarrow \lambda} E_{\mu}$, we finally obtain $\left(I-F_{\lambda}\right) X E_{\lambda}=0$. Now, since $\mathcal{B}_{A, B}$ is weakly closed, it follows from Theorem 2.1 that it is reflexive. 
Now let $\mathcal{N}_{1}$ and $\mathcal{N}_{2}$ be nests on the Hilbert spaces $\mathcal{H}_{1}$ and $\mathcal{H}_{2}$ and let $\mathcal{A}=$ $\operatorname{Alg} \mathcal{N}_{1}, \mathcal{B}=\operatorname{Alg} \mathcal{N}_{2}$ be the corresponding nest algebras. If $\mathcal{U}$ is a weakly closed $\mathcal{B}, \mathcal{A}$-bimodule, then, by Theorem $2.1, \mathcal{U}=\mathrm{Op} \psi$ for some left-continuous mapping $\psi: \mathcal{N}_{1} \longrightarrow \mathcal{N}_{2}$. By [5], Theorem 3.3, $\psi$ extends to a nest mapping $\varphi: \mathcal{P}_{1} \longrightarrow \mathcal{P}_{2}$. Let $\mathcal{L}_{1}$ and $\mathcal{L}_{2}$ be the semi-lattices corresponding to the mapping $\psi$ and let $\widetilde{\mathcal{L}_{1}}$ and $\widetilde{\mathcal{L}_{2}}$ be their completions together with the identity and the zero elements (that is, the smallest nests, containing them). Define $\mathcal{A}_{1}$ to be the set of all positive invertible contractions $A$, having the following two properties:

(i) $A$ has spectral nest equal to $\widetilde{\mathcal{L}_{1}}$,

(ii) if $E \in \widetilde{\mathcal{L}_{1}}$ is such that $\varphi(E)_{+} \notin \mathcal{L}_{2}$, then $A$ has a jump at $E$.

We recall that, for a nest $\mathcal{N}$ and an element $L \in \mathcal{N}, L_{-}=\bigvee\{P \in \mathcal{N}: P<L\}$ and $L_{+}=\bigwedge\{P \in \mathcal{N}: P>L\}$. Now we prove the converse of Theorem 2.2

Theorem 2.3. Let $\mathcal{U}$ be a weakly closed $\mathcal{B}, \mathcal{A}$-bimodule. For every operator $A \in$ $\mathcal{A}_{1}$, there exists a positive invertible contraction $B_{A}$ with a spectral nest equal to $\widetilde{\mathcal{L}_{2}}$, such that $\mathcal{U}=\mathcal{B}_{A, B_{A}}$.

Proof. We recall that $\mathcal{U}=\operatorname{Op} \varphi$ where $\varphi: \mathcal{L}_{1} \longrightarrow \mathcal{L}_{2}$ is a bijection. Let $A \in \mathcal{A}_{1}$ have spectral nest $\mathcal{E}_{A}=\left\{E_{\lambda}\right\}$. First we define the spectral nest of $B_{A}$. Let $0 \leq \lambda \leq 1$. If $\lambda$ is such that $\varphi\left(E_{\lambda}\right)_{+} \in \mathcal{L}_{2}$, then define $F_{\lambda}=\varphi\left(E_{\lambda}\right)$. If $\varphi\left(E_{\lambda}\right)_{+} \notin \mathcal{L}_{2}$, then, by the defenition of the class $\mathcal{A}_{1}, E=E_{\lambda}$ is a jump for $A$. Let $\tau=\left\{\lambda: E=E_{\lambda}\right\}$. $\tau$ is an interval and since for every $\mu, E_{\mu}=\lim _{\nu \downarrow \mu} E_{\nu}$, it is closed from the left. Because $\varphi(E)_{+} \notin \mathcal{L}_{2}$, we have $\left(\varphi(E)_{+}\right)_{+}=\varphi(E)_{+}$and so $E=E_{+}$. This obviously means that $\tau$ is closed also from the right, say $\tau=[a, b]$. If $a \leq \lambda<b$, we define $F_{\lambda}=\varphi\left(E_{\lambda}\right)=\varphi(E)$. If $\lambda=b$, we let $F_{\lambda}=\varphi(E)_{+} \cdot\left\{F_{\lambda}\right\}$ is a spectral nest, so we may define $B_{A}=\int_{0}^{1} \lambda d F_{\lambda}$. From the definition of $\left\{F_{\lambda}\right\}$ it is obvious that

$$
\mathrm{Op} \varphi=\left\{X \in \mathcal{L}\left(\mathcal{H}_{1}, \mathcal{H}_{2}\right):\left(I-F_{\lambda}\right) X E_{\lambda}=0,0 \leq \lambda \leq 1\right\},
$$

so from Theorem 2.2 it follows that $\mathcal{U}=\mathcal{B}_{A, B_{A}}$.

\section{Submodules, the objects $\mathcal{R}_{A}$, the "RAdical"}

In this section, given a $\mathrm{w}^{*}$-closed nest algebra bimodule $\mathcal{U}$, we define a norm closed submodule $\mathcal{R}$ of $\mathcal{U}$, which coincides with the radical when the two algebras and the bimodule are equal. We characterize this object in a number of ways, which generalize known facts about nest algebras. We identify the maximal $\mathrm{w}^{*}$ closed sub-bimodules of the given one, and prove that their intersection coincides with the $\mathrm{w}^{*}$-closure of $\mathcal{R}$.

Definition 3.1. Let $\tau$ be a toplology on $\mathcal{B}\left(\mathcal{H}_{1}, \mathcal{H}_{2}\right), \mathcal{U}$ a $\tau$-closed $\mathcal{B}, \mathcal{A}$-bimodule and $\mathcal{S} \subset \mathcal{U}, \mathcal{S} \neq \mathcal{U}$ a $\tau$-closed sub-bimodule. $\mathcal{S}$ will be called a maximal $\tau$-closed sub-bimodule of $\mathcal{U}$, if the only $\tau$-closed sub-bimodule properly containing $\mathcal{S}$ is $\mathcal{U}$.

Now let $\mathcal{L}_{1}$ be a meet-complete totally ordered set containing the identity, let $\mathcal{L}_{2}$ be a join-complete totally ordered set containing the zero element and let $\varphi$ : $\mathcal{L}_{1} \longrightarrow \mathcal{L}_{2}$ be an order-preserving bijection. Let $\mathcal{N}_{1}=\widetilde{\mathcal{L}}_{1}, \mathcal{N}_{2}=\widetilde{\mathcal{L}}_{2}, \mathcal{A}=\operatorname{Alg} \mathcal{N}_{1}$ and $\mathcal{B}=\operatorname{Alg} \mathcal{N}_{2}$. We will denote by $\psi: \mathcal{L}_{2} \longrightarrow \mathcal{L}_{1}$ the inverse of $\varphi$; let $\mathcal{U}=\operatorname{Op} \varphi$. The following characterization of the rank one operators in $\mathcal{U}$, which is a part of Lemma 6.3 from [5], will be of importance to us. 
Lemma 3.2. Let $R=x \otimes y$ be a rank one operator in $\mathcal{U}$ and let $E$ be the maximal element of $\mathcal{N}_{1}$ with $y \in E^{\perp}$. Then

$$
x \in \begin{cases}\varphi\left(E_{+}\right) & \text {if } E \neq E_{+}, \\ \varphi(E)_{+} & \text {if } E=E_{+} .\end{cases}
$$

Conversely, if the vectors $x$ and $y$ satisfy the above condition for an $E \in \mathcal{N}_{1}$, then the operator $R=x \otimes y$ belongs to $\mathcal{U}$.

Now let $\mathcal{S}$ be a $\mathrm{w}^{*}$-closed $\mathcal{B}, \mathcal{A}$-sub-bimodule of $\mathcal{U}$. By Theorem $2.1, \mathcal{S}=\mathrm{Op} \chi$, where $\chi$ is a left-continuous, order-preserving mapping from $\mathcal{N}_{1}$ to $\mathcal{N}_{2}$. Moreover, $\chi=$ MapS. Because $\mathcal{S} \subset \mathcal{U}$, it follows that $\chi \leq \varphi$. If $\psi$ is a mapping from the class $\mathcal{M}\left(\mathcal{P}_{1}, \mathcal{P}_{2}\right)$, by $\mathcal{L}_{1}(\psi)$ and $\mathcal{L}_{2}(\psi)$ we will denote the semi-lattices corresponding to it. The next theorem identifies the maximal $\mathrm{w}^{*}$-closed sub-bimodules of $\mathcal{U}$.

Theorem 3.3. Let $\mathcal{S} \subset \mathcal{U}$ be a maximal $w^{*}$-closed $\mathcal{B}, \mathcal{A}$-sub-bimodule of $\mathcal{U}$. Then

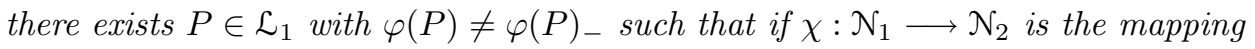

$$
\chi(N)= \begin{cases}\varphi(N) & \text { if } N \neq P, \\ \varphi(N)_{-} & \text {if } N=P,\end{cases}
$$

then $\mathcal{S}=O p \chi$. Conversely, if a mapping $\chi$ has the above form, then the bimodule $\mathcal{S}=O p \chi$ is a maximal $w^{*}$-closed $\mathcal{B}, \mathcal{A}$-sub-bimodule of $\mathcal{U}$.

Proof. Let us first note that because $\varphi(P)_{-} \in \mathcal{L}_{2}\left(\mathcal{L}_{2}\right.$ is closed with respect to unions), and $\varphi$ is a bijection, $\varphi(P) \neq \varphi(P)_{-}$implies that $P \neq P_{-} \in \mathcal{L}_{1}$.

Step 1. We prove that each bimodule of the form $\mathcal{S}=$ Op $\chi$, where $\chi$ is as in the theorem, is a maximal $\mathrm{w}^{*}$-closed sub-bimodule of $\mathcal{U}$. Let us note that $\chi$ is left-continuous, and so by Theorem 4.4 of [5], MapOp $\chi=\chi$. We show that $\mathcal{S} \neq \mathcal{U}$. Let $y \in P \ominus P_{-}$and $x \in \varphi(P) \ominus \varphi(P)_{-}$. Then $R=x \otimes y \in \operatorname{Op} \varphi=\mathcal{U}$. If $P_{0}=\bigvee\left\{Q \in \mathcal{L}_{1}(\chi): y \in Q^{\perp}\right\}$, then $P_{0} \leq P_{-}$. Because $P \in \mathcal{L}_{1}(\chi)$ and $P \neq P_{-}$, from Lemma 3.2 it follows that, in order for $R$ to belong to Op $\chi$ it is necessary that $x \in \chi(P)=\varphi(P)_{-}$, which does not happen. So $R \notin \mathrm{Op} \chi$, and $\mathcal{S} \neq \mathcal{U}$.

Now let $\mathcal{S} \subseteq \mathcal{J} \subseteq \mathcal{U}, \mathcal{J}=O$ Op $\chi_{0}$ for some $\chi_{0}: \mathcal{N}_{1} \longrightarrow \mathcal{N}_{2}$, which is orderpreserving and left-continuous. If $\chi_{0}(P)<\varphi(P)$, then $\chi_{0}(P) \leq \chi(P)$ and so $\mathcal{J} \subseteq \mathcal{S}$. If $\chi_{0}(P)=\varphi(P)$, then $\mathcal{J}=\mathcal{U}$. (Indeed, if $T \in \mathcal{U}$, we have that $\left(I-\chi_{0}(P)\right) T P=0$ from the identity $\chi_{0}(P)=\varphi(P)$ and $\left(I-\chi_{0}(E)\right) T E=0$ for $E \neq P$ because for such $E, \varphi(E)=\chi(E)=\operatorname{MapOp} \chi(E) \leq \operatorname{MapOp}_{0}(E)=\chi_{0}(E) \leq \varphi(E)$, which of course means that $\chi_{0}(E)=\varphi(E)$.)

So we proved that there is no $\mathcal{J}$ between $\mathcal{S}$ and $\mathcal{U}$ and this, together with the fact that $\mathcal{S} \neq \mathcal{U}$, implies that $\mathcal{S}$ is maximal. We will denote by $\mathcal{S}_{P}$ the maximal sub-bimodule which corresponds to a projection $P$ with the stated properties.

Step 2. Let $\mathcal{S}$ be a submodule of $\mathcal{U}$ and let $\mathcal{S}=$ Op $\chi$ for some order-preserving, left-continuous mapping $\chi: \mathcal{N}_{1} \longrightarrow \mathcal{N}_{2}$ and suppose that there exists $P \in \mathcal{L}_{1}$ with $\chi(P)<\varphi(P)_{-}$. We show that then $\mathcal{S}$ is not maximal. We choose $Q \in \mathcal{L}_{2}$ such that $\chi(P) \leq Q<\varphi(P)_{-}$and define two mappings $\chi_{1}, \chi_{2}: \mathcal{N}_{1} \longrightarrow \mathcal{N}_{2}$ as follows:

$$
\begin{aligned}
& \chi_{1}(E)= \begin{cases}\varphi(P)_{-} & \text {if } E=P, \\
\varphi(E) & \text { if } E \neq P,\end{cases} \\
& \chi_{2}(E)= \begin{cases}Q & \text { if } E=P, \\
\varphi(E) & \text { if } E \neq P .\end{cases}
\end{aligned}
$$

If $\mathcal{S}_{1}=\mathrm{Op} \chi_{1}$ and $\mathcal{S}_{2}=\mathrm{Op} \chi_{2}$, then $\mathcal{S} \subseteq \mathcal{S}_{2} \subseteq \mathcal{S}_{1} \subseteq \mathcal{U}$. We show that $\mathcal{S}_{2} \neq \mathcal{S}_{1} \neq \mathcal{U}$. For this, choose $y \in P \ominus P_{-}, x_{1} \in \varphi(P) \ominus \varphi(P)_{-}$and $x_{2} \in \varphi(P)_{-} \ominus Q$. Using 
Lemma 3.2 , it is not difficult to show that $x_{1} \otimes y$ is in $\mathcal{U}$ but not in $\mathcal{S}_{1}$ and $x_{2} \otimes y$ is in $\mathcal{S}_{1}$ but not in $\mathcal{S}_{2}$. Thus, $\mathcal{S}$ is not maximal and we have that $\varphi(P)_{-} \leq \chi(P)$ for each $P \in \mathcal{L}_{1}$.

Step 3. We prove that if $\mathcal{S}=\mathrm{Op} \chi$ is maximal, then there are no more than one $P \in \mathcal{N}_{1}$ such that $\chi(P)=\varphi(P)_{-} \neq \varphi(P)$. Indeed, if there exist $P_{1} \neq P_{2}$ with this property, then, if $\mathcal{S}_{i}=\mathcal{S}_{P_{i}}$ for $i=1,2$, then $\mathcal{S} \subseteq \mathcal{S}_{1,2} \stackrel{\text { def }}{=} \mathcal{S}_{P_{1}} \cap \mathcal{S}_{P_{2}}$. It is easy to check that $S_{1,2} \neq \mathcal{S}_{P_{1}} \neq \mathcal{U}$, which is a contradiction. So there exists exactly one $P \in \mathcal{L}_{1}$ such that $\chi(P)=\varphi(P)_{-} \neq \varphi(P)$. This finishes the proof.

Definition 3.4. A projection $P \in \mathcal{L}_{1}$ with the properties cited in the above theorem will be called a gap projection. We recall that the maximal $\mathrm{w}^{*}$-closed sub-bimodule, corresponding to a gap projection $P$ will be denoted by $\mathcal{S}_{P}$.

Now we introduce some notions and objects, which will turn out to play the role of the radical in the case when the two algebras and the bimodule over them coincide. Let $\mathcal{F}$ be the class of all finite subnests $\left\{E_{i}\right\}_{i=0}^{n}$ of $\mathcal{N}$ (we call them finite partitions). We define the set

$$
\mathcal{R}=\left\{T \in \mathcal{U}: \forall \epsilon>0 \exists\left\{E_{i}\right\}_{i=1}^{n} \in \mathcal{F} \text { with }\left\|\Delta \varphi\left(E_{i}\right) T \Delta E_{i}\right\| \leq \epsilon, 1 \leq i \leq n\right\},
$$

where $\Delta E_{i}=E_{i}-E_{i-1}, \Delta \varphi\left(E_{i}\right)=\varphi\left(E_{i}\right)-\varphi\left(E_{i-1}\right)$. Note that we use a condition analogous to the Ringrose condition [12]. It is obvious that $\mathcal{R}$ is a norm closed bimodule over $\mathcal{B}$ and $\mathcal{A}$. If $A \in \mathcal{A}_{1}$ and $B_{A}$ is the corresponding operator found in Theorem 2.3, we set

$$
\mathcal{R}_{A}=\left\{X \in \mathcal{U}:\left\|B_{A}^{n} X A^{-n}\right\| \longrightarrow_{n} 0\right\} .
$$

It is easy to see that $\mathcal{R}_{A}$ is also a norm closed $\mathcal{B}, \mathcal{A}$-bimodule. Let

$$
\mathcal{R}_{0}=\overline{\left\{\mathcal{R}_{A}: A \in \mathcal{A}_{1}\right\}}
$$

the closure being taken in the norm topology. As in [3], we define for $F, G \in \mathcal{N}_{1}, F \leq$ G,

$$
\mathcal{L}_{F, G}=\left\{X \in \mathcal{B}\left(\mathcal{H}_{1}, \mathcal{H}_{2}\right): \varphi(F) X(I-G)=X\right\}, \quad \mathcal{L}_{F}=\mathcal{L}_{F, F}
$$

It is easy to check that $\mathcal{L}_{F, G} \subseteq \mathcal{U}$ and that $\mathcal{L}_{F, G}$ is a norm closed $\mathcal{B}, \mathcal{A}$-bimodule. The following lemma is a simple generalization of Lemma 7 in [3].

Lemma 3.5. (i) If $A \in \mathcal{A}_{1}$ and $A$ has a jump at $E$, then $\mathcal{L}_{E} \subseteq \mathcal{R}_{A}$;

(ii) If $A \in \mathcal{A}_{1}$ and $F<G$, then $\mathcal{L}_{F, G} \subseteq \mathcal{R}_{A}$.

Proof. (i) Let $\left\{E_{\lambda}\right\}$ and and $\left\{F_{\lambda}\right\}$ be the spectral nests of $A$ and $B$, respectively. If $E_{\lambda}=E$ for $\lambda \in(a, b)$, then certainly $F_{\lambda}=\varphi(E)$ for $\lambda \in(a, b)$ and so if $X \in \mathcal{L}_{E}$, $\left\|B^{n} \varphi(E)\right\|=\left\|B^{n} F_{a}\right\| \leq a^{n}$ and $\left\|(I-E) A^{-n}\right\| \leq b^{-n}$, so

$$
\left\|B^{n} \varphi(E) X(I-E) A^{-n}\right\| \leq\left(\frac{a}{b}\right)^{n}\|X\| \rightarrow 0
$$

The proof of (ii) is analogous.

Proposition 3.6. If $R \in \mathcal{R}$, then $\tilde{\mathcal{J}} \stackrel{\text { def }}{=} \lim _{\mathcal{P}} \sum_{i=1}^{n} \varphi\left(F_{i}\right) R \Delta E_{i}$ (where the limit is taken over all finite partitions $\mathcal{P}$ of $\mathcal{N}_{1}$ and $E_{i-1} \leq F_{i} \leq E_{i}$,) exists and $\tilde{\mathcal{T}}=R$.

Proof. A proof for the case $\mathcal{N}_{1}=\mathcal{N}_{2}$ is given in Theorem 6 in [8]. For $R \in \mathcal{R}$, the upper integral $\tilde{\mathcal{U}}(R) \stackrel{\text { def }}{=} \lim _{\mathcal{P}} \sum_{i=1}^{n} \varphi\left(E_{i}\right) R \Delta E_{i}$ exists and equals $R$ because for each partition $\mathcal{P}$, each member of the above sum is equal to $R$. Furthermore,

$$
\left\|\sum \varphi\left(F_{i}\right) R \Delta E_{i}-R\right\| \leq\left\|\sum\left(\varphi\left(F_{i}\right)-\varphi\left(E_{i-1}\right)\right) R \Delta E_{i}\right\|+\left\|\sum \Delta \varphi\left(E_{i}\right) R \Delta E_{i}\right\|
$$


and the sums on the right hand side can be made arbitrarily small because $R \in$ $\mathcal{R}$.

The first relationship we prove between $\mathcal{R}$ and $\mathcal{R}_{0}$ is the following:

Lemma 3.7. $\mathcal{R} \subseteq \mathcal{R}_{0}$.

Proof. For a partition $\mathcal{P}=\left\{E_{i}\right\}_{i=0}^{n}$ of $\mathcal{N}_{1}$ we define $\mathcal{L}(X: \mathcal{P})=\sum_{i=1}^{n} \varphi\left(E_{i-1}\right) X \Delta E_{i}$. If $X \in \mathcal{R}$, then, from Proposition 3.6 it follows that $X=\lim _{\mathcal{P}} \mathcal{L}(X: \mathcal{P})$. Each $\mathcal{L}(X: \mathcal{P})$ is in the span of $\left\{\mathcal{L}_{E_{i}}, \mathcal{L}_{E_{i-1}, E_{i}}\right\}$. In view of Lemma 3.5, it suffices to find an operator in $\mathcal{A}_{1}$ which has a jump at every $E_{i}, i=1,2, \ldots$. One such operator is $A_{1}=A \prod_{i=1}^{n}\left(I-\frac{1}{2} E_{i}\right)$, where $A$ is an arbitrary operator in $\mathcal{A}_{1}$ [3].

We recall that $\mathcal{U}=\operatorname{Op} \varphi$, where $\varphi: \mathcal{L}_{1} \longrightarrow \mathcal{L}_{2}$ is an order-preserving bijection between the semi-lattices $\mathcal{L}_{1}$ and $\mathcal{L}_{2}, \mathcal{L}_{1}$ closed with respect to the intersections and containing the identity, $\mathcal{L}_{2}$ closed with respect to the unions and containing the zero element. Let $\psi: \mathcal{L}_{2} \longrightarrow \mathcal{L}_{1}$ is its inverse. We define

$$
\mathcal{V}=\mathrm{Op} \psi=\left\{Y \in \mathcal{L}\left(\mathcal{H}_{2}, \mathcal{H}_{1}\right):(I-\psi(F)) Y F=0 \text {, for each } F \in \mathcal{L}_{2}\right\} .
$$

Obviously $\mathcal{V}$ is a $\mathrm{w}^{*}$-closed $\mathcal{B}, \mathcal{A}$-bimodule. We define also

$$
v \operatorname{nil}(\mathcal{U})=\{X \in \mathcal{U}: Y X \text { is quasinilpotent for each } Y \in \mathcal{V}\} \text {. }
$$

We note that $Y X$ is an element of the algebra $\mathcal{A}$. Using the fact that for a Banach algebra $\mathcal{C}$

$$
\begin{aligned}
& \{D \in \mathcal{C}: C D \text { is quasinilpotent for each } C \in \mathcal{C}\}= \\
& \{D \in \mathcal{C}: D C \text { is quasinilpotent for each } C \in \mathcal{C}\}(=\operatorname{rad} \mathcal{C}),
\end{aligned}
$$

one can easily verify that $v \operatorname{nil}(\mathcal{U})$ is a $\mathrm{w}^{*}$-closed $\mathcal{B}, \mathcal{A}$-bimodule.

Our next step to Theorem 3.10 is the following:

Lemma 3.8. $\mathcal{R}_{0} \subseteq v \operatorname{nil}(\mathcal{U})$.

Proof. Because $v$ nil $(\mathcal{U})$ is closed in the norm topology, it suffices to show that $\mathcal{R}_{A} \subseteq \mathcal{v}$ nil( $\left.\mathcal{U}\right)$ for each $A \in \mathcal{A}_{1}$. So let $A \in \mathcal{A}_{1}$ and $B=B_{A}$ be its corresponding operator arising from Theorem 2.3. Pick an $X \in \mathcal{U}$ with $\left\|B^{n} X A^{-n}\right\| \longrightarrow 0$. From Erdos' work [3] it follows that in order to prove that $Y X$ is quasinilpotent for each $Y \in \mathcal{V}$, it suffices to show that, for each $Y \in \mathcal{V},\left\|A^{n}(Y X) A^{-n}\right\|$ tends to zero as $n$ tends to infinity. We have

$$
\left\|A^{n}(Y X) A^{-n}\right\| \leq\left\|A^{n} Y B^{-n}\right\|\left\|B^{n} X A^{-n}\right\| .
$$

As can be easily checked,

$$
\mathcal{V}=\left\{Y \in \mathcal{B}\left(\mathcal{H}_{2}, \mathcal{H}_{1}\right):\left(I-E_{\lambda}\right) X F_{\lambda}=0,0 \leq \lambda \leq 1\right\} .
$$

From Theorem 2.2, it follows that

$$
\mathcal{V}=\left\{Y:\left\|A^{n} Y B^{-n}\right\| \text { is bounded for } n=1,2 \ldots\right\} .
$$

Now, because the first member of the product on the right is bounded and the second one tends to zero, it follows that the product tends to zero.

We recall now some well known facts (see, for example, [1]). Let $C^{*}\left(\mathcal{N}_{1}\right)$ be the $C^{*}$-algebra, generated by the projections of the nest $\mathcal{N}_{1}$ and $\mathcal{M}_{\mathcal{N}_{1}}$, be its maximal ideal space. As it is known, $\mathcal{M}_{\mathcal{N}_{1}}$ is homeomorphic to $\operatorname{Hom}\left(\mathcal{N}_{1}, \mathbf{2}\right)$, the space of all order-preserving functions from $\mathcal{N}_{1}$ to $\mathbf{2} \stackrel{\text { def }}{=}\{0,1\}$ and the homeomorphism is given by the restriction of each element of $\mathcal{M}_{\mathcal{N}_{1}}$ to $\left\{P_{N}: N \in \mathcal{N}_{1}\right\}$. For each 
$\alpha \in \mathcal{M}_{\mathcal{N}_{1}}$ let $\mathcal{E}_{\alpha}=\left\{E=P_{1}-P_{2}: P_{1}, P_{2} \in \mathcal{N}_{1}, \alpha(E)=1\right\}$. We define the seminorm $\|T\|_{\alpha}=\inf \left\{\|\varphi(E) T E\|: E \in \mathcal{E}_{\alpha}\right\}$ and we let $I_{\alpha}=\left\{X \in \mathcal{U}:\|X\|_{\alpha}=0\right\}$ be its kernel. The following result will be of importance to us.

Lemma 3.9. Let $T \in \mathcal{U}, \alpha \in \mathcal{M}_{\mathfrak{N}_{1}}$. There exists $C \in \mathcal{V},\|C\| \leq 1$, such that $\left\|(C T)^{n}\right\|_{\alpha}=\|T\|_{\alpha}^{n}$, for each $n=1,2, \ldots$.

Proof. A simple generalization of Lemma 6.5 from [1].

Now we can formulate and easily prove the following theorem, which shows the connection between the objects we have been introducing. If a mapping $\psi$ is defined on a nest $\mathcal{L}$ and $E=P_{1}-P_{2}$, with $P_{1}, P_{2} \in \mathcal{L}$, we set $\psi(E)=\psi\left(P_{1}\right)-\psi\left(P_{2}\right)$.

Theorem 3.10. The following are equivalent for an $X \in \mathcal{U}$ :

(i) $X \in v \operatorname{nil}(\mathcal{U})$;

(ii) $X \in \bigcap\left\{I_{\alpha}: \alpha \in \mathcal{M}_{\mathcal{N}_{1}}\right\}$;

(iii) $X \in \mathcal{R}$;

(iv) $X \in \mathcal{R}_{0}$.

Proof. The direction (iii) $\Rightarrow$ (iv) is Lemma 3.7 and the direction (iv) $\Rightarrow$ (i) is Lemma 3.8.

(i) $\Rightarrow$ (ii). If $\|X\|_{\alpha}>0$ for some $\alpha \in \mathcal{M}_{\mathfrak{N}_{1}}$, then from Lemma 3.9 it follows that there exists $C \in \mathcal{V}$, such that $C X$ is not quasinilpotent.

(ii) $\Rightarrow$ (iii). Let $X$ be such that $\|X\|_{\alpha}=0$ for each $\alpha \in \mathcal{M}_{\mathcal{N}_{1}}$. Then for every $\epsilon>0$ there exists $E_{\alpha} \in \mathcal{E}_{\alpha}$, such that $\left\|\varphi\left(E_{\alpha}\right) X E_{\alpha}\right\|<\epsilon$. The family $\left\{\mathcal{O}_{E_{\alpha}}: \alpha \in \mathcal{M}_{\mathcal{N}_{1}}\right\}$, where $\mathcal{O}_{E}=\left\{\psi \in \mathcal{M}_{\mathcal{N}_{1}}: E \in \mathcal{E}_{\psi}\right\}=\left\{\psi: \psi\left(N_{1}\right)>\frac{1}{2}>\psi\left(N_{2}\right)\right\}$, is an open cover for $\mathcal{M}_{\mathcal{N}_{1}}$; because $\mathcal{M}_{\mathcal{N}_{1}}$ is compact, it follows that there is a finite subcover, say $\left\{\mathcal{O}_{E_{n}}\right\}_{n=1}^{m}$. Now if $\mathcal{P}$ is a partition of $\mathcal{N}_{1}$ with endpoints the endpoints of $E_{n}$, $n=1,2, \ldots, m$, then $\mathcal{P}$ satisfies the Ringrose condition for $X$.

The last theorem will enable us to characterize the intersection of the maximal $\mathrm{w}^{*}$-closed sub-bimodules of a given module (Theorem 3.14), which is the main result of this section. Let

$$
\mathcal{T}=\bigcap\left\{\mathcal{S}: \text { a maximal } \mathrm{w}^{*} \text {-closed sub-bimodule of } \mathcal{U}\right\} .
$$

First we establish the following:

Lemma 3.11. Let $A \in \mathcal{A}_{1}$ and $\mathcal{S}$ be a maximal $w^{*}$-closed sub-bimodule of $\mathcal{U}$. Then $\mathcal{R}_{A} \subseteq \mathcal{S}$.

Proof. In view of Theorem 3.3, $\mathcal{S}$ is of the form $\mathcal{S}=\mathcal{S}_{P}$, for some gap projection $P \in \mathcal{N}_{1}$. Let $P_{-}=E_{\lambda_{0}}, P=E_{\lambda}$, where $\left\{E_{t}\right\}$ is the spectral nest of $A$. If $\left\{F_{t}\right\}$ is the spectral nest of $B=B_{A}$, then obviously $F_{\mu}=F_{\lambda_{0}}=\varphi(P)_{-}$for each $\mu$ with $\lambda_{0} \leq \mu<\lambda$. Hence, we have for $X \in \mathcal{R}_{A}$ and $\mu$ between $\lambda_{0}$ and $\lambda$,

$$
\begin{array}{r}
\left\|\left(I-\varphi\left(P_{-}\right)\right) X P\right\|=\left\|\left(I-F_{\lambda_{0}}\right) X E_{\lambda}\right\|=\left\|\left(I-F_{\mu}\right) X E_{\lambda}\right\| \\
\leq\left\|\left(I-F_{\mu}\right) B^{-n}\right\|\left\|B^{n} X A^{-n}\right\|\left\|A^{n} E_{\lambda}\right\| \leq\left(\frac{\lambda}{\mu}\right)^{n} c_{n},
\end{array}
$$

where $c_{n}=\left\|B^{n} X A^{-n}\right\| \longrightarrow 0$. If $n_{0}$ is such that $1+\frac{1}{n_{0}} \leq \frac{\lambda}{\lambda_{0}}<1+\frac{1}{n_{0}-1}$, we define $\mu_{n}=\lambda /\left(1+\frac{1}{n}\right.$ ) (i.e. $\left.\frac{\lambda}{\mu_{n}}=1+\frac{1}{n}\right)$, for $n \geq n_{0}$. But then $\left(\frac{\lambda}{\mu_{n}}\right)^{n} c_{n} \rightarrow 0$ as $n$ tends to infinity, and hence, $\left(I-\varphi\left(P_{-}\right)\right) X P=0$, which means that $X \in \mathcal{S}$. 
Corollary 3.12. $\overline{\mathcal{R}}^{w *} \subseteq \mathcal{T}$.

Proof. Immediate from the definition of $\mathcal{T}$ and Theorem 3.10.

Our last theorem establishes the equality between the two objects of the last corollary. Let us first note that each (one- or two-sided) nest algebra bimodule $\mathcal{S}$ is $\mathrm{w}^{*}$-generated by the operators of rank one contained in it, in the sense that $\mathcal{S}={\overline{\mathcal{R}_{1}(\mathcal{S})}}^{w *}$, where $\mathcal{R}_{1}(\mathcal{S})$ is the subspace of $\mathcal{S}$ spanned by its rank one operators. This is a consequence of the same fact about nest algebras [4]. We need a little lemma, whose proof is a simple generalization of the proof of Lemma 3.5 of [1].

Lemma 3.13. Let $\varphi$ be a nest mapping and $K$ be a compact operator in $\operatorname{Op} \varphi$. Given $\epsilon>0$, there is a partition $\left\{E_{i}\right\}_{i=1}^{s}$ of $\widehat{\mathcal{L}_{1}(\varphi)}$ so that for each $i=1,2, \ldots, s$ either $E_{i}$ is an atom or $\left\|\varphi\left(E_{i}\right) K E_{i}\right\|<\epsilon$.

Theorem 3.14. $\mathcal{T}=\overline{\mathcal{R}}^{w *}$.

Proof. It remains only to establish the inclusion $\mathcal{T} \subseteq \overline{\mathcal{R}}^{w *}$. Because $\mathcal{T}$ is $\mathrm{w}^{*}$-closed, according to the note above, it suffices to show that each operator of rank one in $\mathcal{T}$ belongs to $\mathcal{R}$. By Theorem 3.3, $\mathcal{T}$ is of the form $\mathcal{T}=$ Op $\chi$, where

$$
\chi(P)=\varphi(P)_{-} .
$$

As was mentioned in the beginning of Theorem 3.3, $\varphi(P) \neq \varphi(P)_{-}$implies $P \neq P_{-}$. So every compact operator $T$ in $\mathcal{T}$ satisfies the Ringrose condition. Indeed, given an $\epsilon>0$, let $\left\{E_{i}\right\}$ be the partition obtained in Lemma 3.13. If $E_{i}$ is not an atom, then $\left\|\varphi\left(E_{i}\right) T E_{i}\right\| \leq \epsilon$ and if $E_{i}$ is an atom, $\varphi\left(E_{i}\right) T E_{i}=0$. So $T$ belongs to $\mathcal{R}$.

Corollary 3.15. The bimodule $\mathcal{U}$ has a maximal $w^{*}$-closed sub-bimodule if and only if $\overline{\mathcal{R}}^{w *} \neq \mathcal{U}$.

Proof. Immediate from Theorem 3.14.

Concluding remark. After the first draft of this paper was completed, we learned that Theorem 2.2 and a weaker version of the Theorem 2.3 were previously proved by Marculescu [10]. The author wishes to thank Dr. J.A. Erdos for providing this information.

\section{ACKNOWLEDGMENT}

The author wishes to express his deepest gratitude to his advisor Aristides Katavolos for his discussions and help during the preparation of this paper.

\section{REFERENCES}

1. K. R. Davidson, Nest algebras. Triangular forms for operator algebras on Hilbert spaces, Longman, 1988. MR 90f: 47062

2. J. A. Deddens, Another description of nest algebras, Hilbert space operators, Lecture Notes in Math. 693, Springer, New York, (1978), 77-86. MR 80f: 47033

3. J. A. Erdos, On some ideals of nest algebras, Proc. London Math. Soc. (3) 44 (1982), 143-160. MR 83g: 47045

4. J. A. Erdos Operators of finite rank in nest algebras, J. London Math. Soc. 43 (1968), 391-397. MR 37:5721

5. J. A. Erdos, Reflexivity for subspace maps and linear spaces of operators, Proc. London Math. Soc. (3) $\mathbf{5 2}$ (1986), 582-600. MR 87h:47103

6. J. A. Erdos and W. E. Longstaff, The convergence of triangular integrals of operators on Hilbert space, Indiana Univ. Math. J. 22 (1973), 929-938. MR 49:1178 
7. J. A. Erdos and S. C. Power, Weakly closed ideals of nest algebras, J. Operator Theory 7 (1982), 219-235. MR 84a:47056

8. S. Karanasios, Triangular integration with respect to a nest algebra module, Indiana Univ. Math. J. 34 (1985), 299-317. MR 86h:47073

9. R. I. Loebl and P. S. Muhly, Analycity and flows in von Neumann algebras, J. Funct. Anal. 29 (1978), 214-252. MR 81h:46080

10. D. I. Marculescu, Reflexivity of linear manifolds of operators, Doctoral thesis, University of London, 1984.

11. M. Radjabalipour, Operators commuting with positive operators, Proc. Amer. Math. Soc. $\mathbf{7 7}$ (1979), 107-110. MR 81d:47014

12. J. R. Ringrose, On some algebras of operators, Proc. London Math. Soc. (3) 15 (1965), 61-83. MR 30:1405

13. A. I. Loginov and V. S. Shulman, Hereditary and intermediate reflexivity of $W^{*}$-algebras, (Russian) Izv. Akad. Nauk. SSSR Ser. Mat. 39 (1975), 1260-1273; English translation, Math. USSR-Izv. 9 (1975), 1189-1201. MR 53:8919

Department of Mathematics, University of Athens, Panepistemioupolis 15784, Athens, GREECE

E-mail address: itodorov@atlas.uoa.gr 